\title{
Special focus issue on regenerative medicine in society: interdisciplinary perspectives (part I) - Foreword
}

\author{
Achim Rosemann ${ }^{*}, 1,2$, Jan Barfoot ${ }^{3} \&$ Clare Blackburn $^{3}$ \\ ${ }^{1}$ Centre for Education Studies, Faculty of Social Sciences, University of Warwick, Coventry, CV4 7AL, UK \\ ${ }^{2}$ Centre for Bionetworking, School of Global Studies, University of Sussex, Brighton, BN1 9SJ, UK \\ ${ }^{3}$ Medical Research Council Centre for Regenerative Medicine, School of Biological Sciences, University of Edinburgh, 5 Little France \\ Drive, Edinburgh, EH16 4UU, UK \\ * Author for correspondence: A.Rosemann@warwick.ac.uk
}

First draft submitted: 15 August 2017; Accepted for publication: 15 August 2017; Published online: 11 October 2017

Keywords: bioethics $\bullet$ ethics $\bullet$ innovation $\bullet$ interdisciplinary $\bullet$ legal $\bullet$ policy $\bullet$ public $\bullet$ regenerative medicine $\bullet$ regulation • social $\bullet$ public engagement $\bullet$ patient needs

During the last two decades, funding bodies in many countries have financed a growing number of interdisciplinary research projects that have examined the social, ethical, regulatory, legal, economic and healthcare aspects of the regenerative medicine field. This emerging body of research has contributed to the shaping and evaluation of regulations and policies, the facilitation of public debate and engagement, and the critical reflection of the practices, limits and values through which new therapeutic strategies are translated from the lab bench to patients, healthcare systems and the market. It has also played a vital role in identifying and discussing the challenges and opportunities that result from the globalization of regenerative medicine research and the existence of regulatory, cultural and socio-economic differences between countries.

This two-part special focus issue brings together articles from current interdisciplinary projects on regenerative medicine innovation, with the aim of communicating key findings to a broad multidisciplinary audience of researchers, clinicians, regulators, policy makers and corporate leaders, as well as patients, patient organizations and interested members of the public. The two issues present insights from various areas of regenerative medicine and from numerous countries and world regions, including Australia, Canada, China, the EU, Germany, France, India, Japan, Mexico, South Korea, Taiwan, the UK and the USA.

The special focus issue is intended to be directly relevant to scientists, clinicians, patients and regulators. It aims to inform public, policy and regulatory debates, at a time where regulatory requirements for regenerative medicine are getting more diverse globally and new (and often contested) methodologies, ethical standards, marketing practices and forms of clinical evidence are entering mainstream clinical medicine. It also aims to contribute to medicine and bioethics curricula in universities and professional training. It is our hope that the articles collated in this double issue offer new insights for educational discussion, the development of relevant case studies, and the transmission of up-to-date knowledge on the social, cultural and regulatory dimensions and trends that shape research and clinical applications in regenerative medicine.

Alongside Achim Rosemann, the European Commission-funded EuroStemCell project has collaborated with Regenerative Medicine as a co-guest editor of this two-part special focus issue, through the involvement of Jan Barfoot and Clare Blackburn. The EuroStemCell project works to provide accessible, trusted and high-quality information on stem cell research to citizens and stakeholders across Europe. The actions of the project are supported by a strong and growing network of scientific and scholarly partners who help ensure the work of the project is both credible and up-to-date. The goals of the project are twofold: to help people make sense of stem cell research and to help the stem cell scientific community make sense of public engagement.

Due to the unique interdisciplinary nature of this two-part special focus issue and the imperative to facilitate broad access to the ideas and findings contained within these issues published in Regenerative Medicine, we are also publishing a free-to-access layperson's summary of each article on the EuroStemCell website (www.eurostemcell.org) 
and the RegMedNet website (www.regmednet.org). We invite readers to share these free-to-access summaries with relevant stakeholders in national and international contexts.

\section{Regenerative medicine in society: interdisciplinary perspectives - part I}

Part I of this special focus issue (Part II will be published in the October issue of Regenerative Medicine) opens with three commentary pieces on recent regulatory developments.

Linda Hogle and Amritava Das open with a commentary on the social production of evidence in the regenerative medicine field by reflecting on the impact of the passing of the 21st Century Cures Act and a growing number of Right-To-Try laws (at the state level) in the USA [1]. The authors state that these laws precipitate fundamental changes to regulatory frameworks for regenerative medicine, and alter the ways in which evidence may be collected and assessed. Most importantly, these changes provide the institutional means to use alternative sources of evidence and information, a step that disturbs long-held beliefs about what counts as credible data and methodological procedures through which evidence is generated.

In the next commentary, Jennifer Liu problematizes what counts as 'good' and 'bad' in regenerative medicine and stem cell science [2]. By referring to examples of controversial technology applications in the USA, China, Taiwan and Mexico, she encourages readers to go beyond dominant notions of the 'good' in science and to take into account the complex social and relational factors that shape normative considerations across the globe. A one-size-fits-all ethics in regenerative medicine that imposes a unified view of 'good science' neglects the plurality of socio-cultural contexts, healthcare priorities and moral views through which medical and clinical research practices come into being. As a solution Liu calls for a 'situated stem cell ethics' that takes seriously the varied terrain in which new medical practices emerge and that shows 'greater appreciation for the variability in ethical prioritization' that exist in the world.

Haidan Chen's commentary reflects on the governance of clinical stem cell research and applications in China [3]. The People's Republic of China has for many years played an important role in regenerative and stem cell medicine, but the development of a consistent regulatory framework for clinical applications has been surprisingly slow and difficult to achieve. Chen suggests that these problems are rooted in a tension in the overall governance structure in China, in particular the tension between the centralization of authority and effective governance at the level of local governments across China's dispersed geographic regions. Chen comments that only by resolving this tension regenerative stem cell research can realize its full potential in China and deliver formally approved and internationally marketable treatments.

In a research article, Christine Hauskeller examines the practical implications of regulatory harmonization for regenerative and stem cell medicine in the EU [4]. Reporting findings from a sociological study of a Phase III multi-country clinical trial, Hauskeller discusses the multiple obstacles that recent harmonized regulation creates for multinational European trials. The article presents a typology of identified obstacles that shall help other research teams to adequately plan and anticipate some of the challenges they are likely to encounter.

Reimbursement of new medical products is one of the key issues in drug development because it has an impact on economic profits and determines who is able to access new treatments. The research article from Aurelie Mahalatchimy's and Alex Faulkner investigates the trends and central institutional dynamics that constitute the policies for reimbursement of regenerative medicine, in the context of the UK [5]. By analyzing the publications, policies and politics of regenerative medicine reimbursement, they show that the UK has still a long way to go to solve the reimbursement challenge for regenerative medicine products. While trade organizations have developed various proposals to solve reimbursement issues for regenerative medicine, there appears a need for more collaboration between several national-level key stakeholders.

Katherine Bonter and colleagues then provide an overview of and analyze trends in the global landscape of clinical trials for cellular immunotherapies [6]. The article identifies opportunities and challenges to aid developers, investors, adopters and payers in this sector to plan for the implementation of clinical trials along realistic time horizons. Overall, trends point to an increasingly personalized approach to onco-immunology. This raises various challenges for cost-effective manufacturing and delivery models.

Hung-Chieh Chang provides a special report that discusses the use, banking and circulation of human cord blood in China [7]. Chang explores how the interplay of state-led planning and capitalism have facilitated the domestic use of cord blood in China, but at the same time have prevented cord blood from being moved across national borders. The article provides an informative overview of China's current landscape of cord blood banking, key policies and the networks and strategies that are related to the circulation and clinical use of cord blood. 
In a perspective piece focused on China, Joy Zhang examines recent initiatives to promote research accountability in the context of the country's stem cell governance [8]. By focusing on regulatory developments in the last fifteen years, Zhang shows that the government's 'post hoc pragmatism' policy rationale, which has characterized the governance of stem cell research in recent years, has harmed rather than benefited medical progress in the stem cell field. She concludes that policy makers need to get beyond an 'act-in-response' regulatory ethos and engage with diverse stakeholders, of they want to improve China's accountability and international standing in regenerative medicine.

Tamra Lysaght shares her perspective on the acceleration of the market approval process for regenerative and cellular medicines in the aftermath of a recent regulatory reform in another East Asian country: Japan [9]. Lysaght suggests that Japan's accelerated approval process raises ethical challenges that have not yet been fully explored. Her paper reflects on these challenges from three perspectives: public trust in science and medicine, distributive justice and the prioritization of safety over clinical benefit.

Gene editing of human gametes, zygotes and embryos has become a widely debated topic in the last years. With the development of CRISPR gene-editing technologies, the prospect of genetically modified offspring has come within close reach. In this special issue, Inigo de Miguel Beriain examines the ethical and legal debates and the current regulatory framework for human embryo and germ line gene editing in the EU [10]. De Miguel Beriain suggests that the legal framework for the use of CRISPR technologies on human gametes, embryos and early fetuses is in urgent need of an update. Considering the rapid developments in this technology field regulators should also prepare for the pending issue of clinical applications, especially if public opinion changes and calls for the reproductive use of this technology are getting louder.

Dusko Ilic and colleagues discuss the ethical and quality considerations of the creation of human embryos from iPS cell-derived gametes [11]. The derivation of human preimplantation embryos from 'artificial' (i.e., pluripotent stem cell-derived) gametes is now technically feasible and could solve the scarcity of human cleavage stage embryos donated for research. However, there are various technological and ethical concerns related to the donation and use of these embryos for research purposes. The article provides an overview of these concerns and proposes a range of technical standards and norms that would assure the quality and reliability of such embryos.

The article from Michael Morrison and colleagues examines the implications of the new European General Data Protection Regulations, which comes into effect in 2018, for iPS cell researchers and iPS cell biobanks [12]. The authors identify the challenges for the iPS cell field that result from this regulation and offer recommendations for the development of appropriate governance structures to ensure compliance with the requirements of the forthcoming change in data protection law.

Leigh Turner's article examines how US businesses that advertise unapproved for-profit stem cell interventions use the NIH clinical trials registry and database ClinicalTrials.gov to recruit patients for 'pay-to-participate' clinical studies [13]. While most of these businesses appear to operate in a legal grey area and their clinical services have not been approved by the FDA or other national regulatory bodies (see also [14]), the use of ClinicalTrials.gov seems to have evolved to a marketing device to sell experimental stem cell interventions to patients. Turner suggests that the growing inclusion of such studies in ClinicalTrials.gov reveals that the NIH registry needs better screening tools, to make sure that the integrity and utility of the database is not compromised and that there is a clear distinction between profit-oriented experimental interventions and clinical research.

\section{Conclusion}

It is our hope that readers will enjoy these articles and be stimulated to engage critically with ideas and concepts about regenerative medicine from disciplines beyond their normal field of work. It is our further intention and aspiration that this body of work will be accessed by global policymakers, regulators, funders and others such as healthcare managers to generate relevant new conversations in their sectors and directly or indirectly inform future decisions regarding the regenerative medicine field in its broadest sense.

\section{Financial \& competing interests disclosure}

The work on this special focus issue has benefitted from research support provided by the ERC (283219), the ESRC (ES/I018107/1) and the Wellcome Trust (204799/Z/16/Z). EuroStemCell receives funding from the EU's Horizon 2020 research and innovation programme under grant agreement No 652796. It has previously been funded under the European Union's Framework 6 and Framework 7 funding streams under grant agreements 503005 and 241878, respectively. The authors have no other 
relevant affiliations or financial involvement with any organization or entity with a financial interest in or financial conflict with the subject matter or materials discussed in the manuscript apart from those disclosed.

No writing assistance was utilized in the production of this manuscript.

\section{References}

1 Hogle LF, Das A. The social production of evidence: regenerative medicine and the 21st Century Cures Act. Regen. Med. 12(6), 581-586 (2017).

2 Liu JA. Situated stem cell ethics: beyond good and bad. Regen. Med. 12(6), 587-591 (2017).

3 Chen H. Reflection on the governance of clinical stem cell research and applications in China. Regen. Med. 12(6), 593-597 (2017).

4 Hauskeller C. Can harmonized regulation overcome intra-European differences? insights from a European Phase III Stem Cell Trial. Regen. Med. 12(6), 599-609 (2017).

5 Mahalatchimy A, Faulkner A. The emerging landscape of reimbursement of regenerative medicine products in the UK: publications, policies and politics. Regen. Med. 12(6), 611-622 (2017).

6 Bonter K, Breckenridge Z, Lachance S, Delisle JS, Bubela T. Opportunities and challenges for the cellular immunotherapy sector: a global landscape of clinical trials. Regen. Med. 12(6), 623-636 (2017).

7 Chang HC. The role of policies and networks in development of cord blood usage in China. Regen. Med. 12(6), 637-645 (2017).

8 Zhang JY. Lost in translation? Accountability and governance of clinical stem cell research in china. Regen. Med. 12(6), 647-656 (2017).

9 Lysaght T. Accelerating regenerative medicine: the Japanese experiment in ethics and regulation. Regen. Med. 12(6), 657-668 (2017).

10 De Miguel Beriain I. Ethical and legal issues regarding gene editing at the beginning of life: an EU perspective. Regen. Med. 12(6), 669-679 (2017).

11 Ilic D, Ogilvie C, Noli L, Kolundzic N, Khalaf Y. Human embryos from iPSC-derived gametes: ethical and quality considerations. Regen. Med. 12(6), 681-691 (2017).

12 Morrison M, Bell J, George C, Harmon S, Munsie M, Kaye J. The European general data protection regulation: challenges and considerations for iPSC researchers and biobanks. Regen. Med. 12(6), 693-703 (2017).

13 Turner L. ClinicalTrials.gov, stem cells, and pay-to-participate clinical studies. Regen. Med. 12(6), 705-719 (2017).

14 Turner L, Knoepfler P. Selling stem cells in the USA: assessing the direct-to-consumer industry. Cell Stem Cell. 19(2), 154-7 (2016). 